\title{
The role of visual experience in knowledge of spatial layout
}

\author{
JOHN J. RIESER \\ Vanderbilt University, Nashville, Tennessee 37203 \\ and \\ JEFFREY J. LOCKMAN and HERBERT L. PICK, JR. \\ University of Minnesota, Minneapolis, Minnesota 55455
}

\begin{abstract}
Adventitiously blinded, congenitally blind, and sighted adults made relative distance judgments in a familiar environment under three sets of instructions-neutral with respect to the metric of comparison, euclidean (straight-line distance between landmarks), and functional (walking distance between landmarks). Analysis of error scores and multidimensional scaling procedures indicated that, although there were no significant differences among groups under functional instructions, all three groups differed from one another under euclidean instructions. Specifically, the sighted group performed best and the congenitally blind group worst, with the adventitiously blind group in between. The results are discussed in the context of the role of visual experience in spatial representation and the application of these methods for evaluating orientation and mobility training for the blind.
\end{abstract}

Studies of early sensory deprivation have examined the role played by various classes of sensory experience in perceptual development. For example, animals have been reared in the dark and in the absence of self-produced locomotion to determine the roles played by visual afference and reafference on the development of depth perception and perceptual-motor coordination (e.g., Gibson \& Walk, 1960; Hein \& Diamond, 1972). The behavior of congenitally blind adults with restored vision has been used to infer the role of prior visual experience in the visual perception of shape, particularly the visual identification of shapes previously known by touch (e.g., see Gregory \& Wallace, 1963; Hebb, 1937).

In contrast with this concentration on the perceptual aspects of spatial development, relatively little research has been concerned with the role of visual experience in the representation and retrieval of spatial information. That people have spatial knowledge is shown by the fact that they can find their way around a space,

The research reported here was supported by Program Project Grant HD 050207 from the National Institutes of Health to the Institute of Child Development of the University of Minnesota and by the Center for Research in Human Learning of the University of Minnesota. The work of the Center is supported by research grants from the National Science Foundation and from the National Institute of Child Health and Human Development. Preparation of the manuscript was also supported by National Institutes of Health Grant HD 04510 to Vanderbilt University through its George Peabody College for Teachers. Many thanks are due to the people who served as subjects in the study and to Gordon Oien and the Mobility Staff at the Minneapolis Society for the Blind. We wish to thank Phipps Arabie for technical assistance rendered. as they demonstrate by taking a route from one place to another. Such route knowledge has been considered a relatively primitive level of spatial knowledge (Hazen, Lockman, \& Pick, 1978; Siegel \& White, 1975); it could be based on proprioceptive or visual information but does not go beyond the information directly available while walking from place to place in order to explore the space. A second, more sophisticated level of spatial knowledge is layout knowledge, reflected in what Siegel and White (1975) refer to as survey-type cognitive maps. This level of knowledge is characterized by easy retrieval of the spatial relations among all pairs of locations, independent of the experienced routes between them. Thus, persons with layout knowledge know the euclidean distances and directions between locations even when those locations are out of sight behind barriers or walls, or are too far away to be seen. With such layout knowledge, it should be easy to find novel routes and make detours.

What is the role of visual experience in determining the level of one's spatial knowledge? A distinction should be made between prior visual experience in general and specific visual experience with the space in question. For example, the complete absence of visual experience during one's life, as in the case of congenitally blind persons, may result in a way of approaching spatial problems and processing spatial information different from that used by persons who have had visual experience. Whatever the case with such general visual experience, it may be that the presence or absence of visual experience with a specific space is an important determiner of how that space is mentally represented. 
Since route knowledge is directly available from both visual and proprioceptive (including kinesthetic and motor) sources, the presence or absence of either specific or general visual experience is probably not important at this level of spatial knowledge. Indeed, blind persons do find their way around their world. On the other hand, the total absence of prior visual experience may affect the representation of spatial layout information. In the first place, vision provides information about the spatial relations among locations to a much greater degree than any other sense modality. We can see many relations directly, for example, whether location $A$ is on a line between $B$ and $C$ or closer to $D$ than to that line. The spatial layout of locations is in many cases directly given in vision. Given such direct experience with visible layout when one has had visual experience, it may be a relatively easy step to extrapolate this representation (e.g., see Attneave \& Farrar, 1977). In the second place, when one moves about the world with vision, one can literally see the simultaneous transformations of the directions of objects from oneself. Thus, heading toward $A$ with $B$ ahead on the right and $C$ ahead on the left, one sees that the direction of $B$ becomes progressively farther to the right and the direction of $\mathrm{C}$ farther to the left. If a wall gradually occludes the view of $B$, it may be relatively easy to extrapolate the changing direction of $B$ relative to oneself, even though it is now out of sight. A lifetime of such experience may alter the representation of spatial layout even when the layout was never seen.

Specific visual experience may also be necessary for representing layout information. Although this level of spatial knowledge involves representation of the relations of locations out of sight of each other, there is visual continuity in moving from one such location to another. Even if specific visual experience is not necessary for such representation, it may be helpful.

The present study presents a method for addressing such issues by comparing adventitiously blinded, congenitally blind, and sighted subjects. In the study, subjects of these three types were asked to make comparative distance judgments among pairs of locations in a very familiar space. One question was whether such judgments conform more closely to the euclidean, or straight-line, distances between the locations or to the functional, or walking, distances. A second question concerns the degree to which subjects can be flexible in use of their spatial knowledge, that is, whether they can easily shift from judging distances on a euclidean to a functional basis when it is efficient to do so. All the subjects in this study knew the space on the basis of proprioceptive, kinesthetic, and motor information, and the sighted subjects kriew the space visually as well. If the sighted and adventitiously blinded subjects produced results similar to each other's but different from those of the congenitally blind subjects, a reasonable interpretation would be that specific visual experience with a space is not an important factor but general prior visual experience is. If the results of the sighted subjects differ from those of the two blind groups, which are similar to each other, the interpretation would be that specific visual experience is important.

\section{METHOD}

\section{Subjects and Experimental Space}

Twelve adults served as subjects-four sighted, four adventitiously blinded, and four congenitally blind. The adventitiously blinded subjects ranged in age from 24 to 60 years and had shown light perception or less vision for at least 3 years. The congenitally blind subjects ranged in age from 21 to 35 years and showed light perception or less vision. The sighted subjects ranged in age from 23 to 40 years. All the subjects were quite familiar with the experimental space, since they had traveled it daily for at least several months and could travel readily from each landmark location to every other location without the benefit of vision. The four sighted subjects worked as instructors of mobility at the Minneapolis Society for the Blind. The eight blind subjects were clients of the society; each had undergone orientation and mobility training for more than 10 weeks before the experimental tests. The pool of such highly experienced and competent subjects was necessarily small.

The experimental space used in the study was the Rehabilitation Section of the Minneapolis Society for the Blind, an area traveled daily by each subject. Within this area, a set of 15 landmark locations, mostly classroom doorways, was selected (these are depicted in Figure 2a). Travel between some locations could be accomplished by walking a straight line, whereas travel between other locations was blocked by walls or other obstacles and involved routes with turns.

\section{Design and Procedures}

The subjects were tested individually, during three sessions of 1 to $2 \mathrm{~h}$ each. Distance judgments were collected using the method of triadic comparisons. Three locations were named and the subjects were asked to identify the two that were closest together and the two farthest apart. In order to reduce the 455 possible triads of 15 locations to a more manageable size, a balanced subset of 140 triads was selected following a procedure developed by Levelt, Van de Geer, and Plomp (1966). ${ }^{1}$

The distance judgments were made by the congenitally blind, adventitiously blinded, and sighted subjects under three sets of instructions: (a) neutral-the subjects were simply asked to perform the triadic comparisons in the easiest, most efficient manner, (b) euclidean-the subjects were asked to base their triadic comparisons on the straight-line distances between locations, or (c) functional-the subjects were asked to base their triadic comparisons on the shortest functional distances between locations. All the subjects completed the comparisons under the three sets of instructions. Judgments were collected first under the neutral instructions. This type of instruction was designed to allow the subjects' spontaneous judgments to be contrasted with their judgments when their attention was focused on a specific metric of comparison, as was the case during the latter two sessions. The order in which the subjects were instructed to base their judgments on euclidean and functional metrics was counterbalanced within each subject group.

\section{RESULTS AND DISCUSSION}

Scores were assigned to the subjects' triadic comparisons as follows: The pair judged closest together 
was assigned the value 2 , the pair judged farthest apart was assigned a 0 , and the remaining, intermediate pair was assigned a 1. Each pair was judged in the context of four different triads (cf. Levelt et al., 1966). Under each set of instructions, the four judgments of each subject per location pair were totaled. These scores were analyzed in three ways. For the first analysis, the accuracy of each subject's judgments was computed relative to two actual measures of the space, a straight-line measure and a functional measure taken from a blueprint of the experimental space. An errorless set of triadic comparison judgments (the performance of a hypothetical ideal euclidean or functional subject) was then derived from those measures. The error score was the sum of the absolute differences between each subject's distance judgments and the errorless distance judgments. These error scores were subjected to analyses of variance.

The second analysis consisted of partial correlations between the judgments of the various subject groups under different instructions with the judgments of the hypothetical ideal euclidean or functional subject.

For the third analysis, each group's judgments under the straight-line instruction condition were represented spatially, so that their judgments could be graphically related to actual properties of the spatial layout of the experimental space. This was accomplished by using multidimensional scaling procedures.

\section{First Analysis: Error Scores}

Error scores were compared for the three groups of subjects in two 3 (subject group) by 2 (instruction) analyses of variance.

In the first, errors were relative to the performance of the ideal euclidean observer; in the second, errors were relative to the performance of the ideal functional observer. The means of these error scores appear in Figure 1. For the euclidean errors, the analysis of variance indicated significant group differences $[F(2,9)$ $=22.4, p<.01]$ and a significant interaction $[\mathrm{F}(2,9)$ $=4.68, \mathrm{p}<.05]$. Since the interaction was significant, tests of the simple effects of subject group were carried out under each set of instructions. All three groups differed from each other under euclidean instructions ( $p<.05$, Tukey test) but did not differ under neutral instructions. For the functional errors, the analysis of variance indicated no significant differences. Thus, the sighted group performed best, the congenitally blind group worst, and the adventitiously blinded group in between under euclidean instructions. In contrast, all groups performed at the same level of accuracy under functional instructions.

\section{Second Analysis: Partial Correlations}

The purpose of this analysis was to relate the sub-
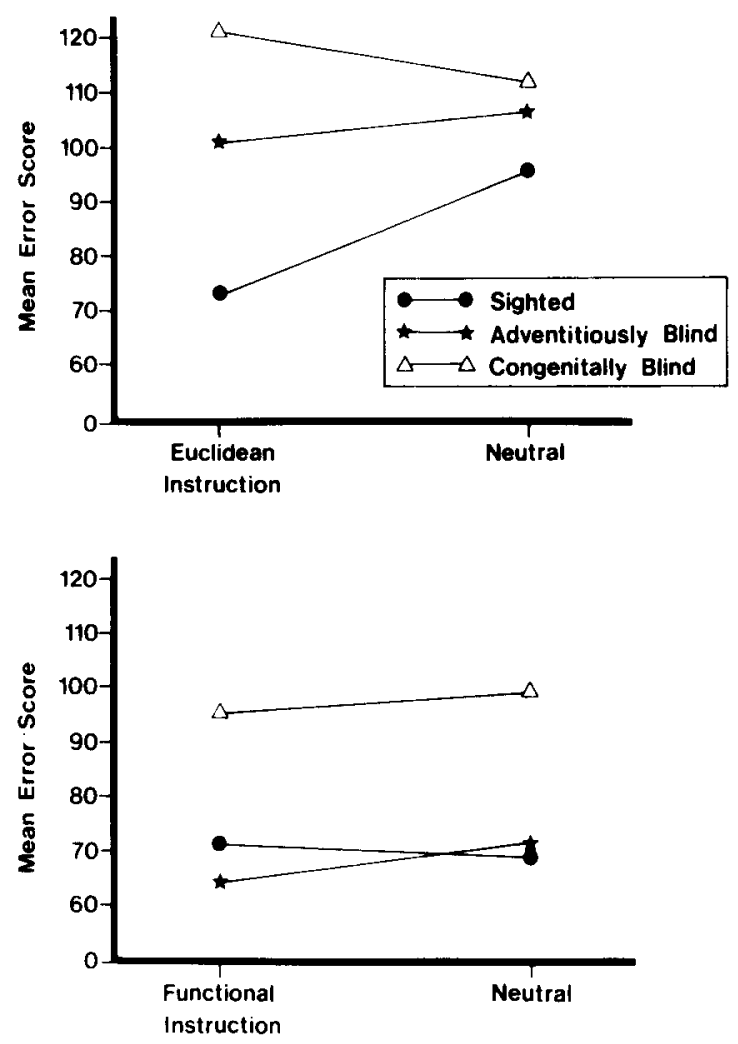

Figure 1. Mean error scores as a function of instruction for sighted, adventitiously blinded, and congenitally blind groups.

jects' distance judgments to those predicted for an ideal euclidean or functional subject. This was done for the judgments obtained under neutral instructions in order to determine the subjects' natural metric for making distance judgments. Partial correlations were also obtained between the subjects' judgments under euclidean instructions with the judgments of the ideal euclidean subject and between the judgments under functional instructions with the judgments of the ideal functional subject, to determine how accurate the subjects were when asked to respond in terms of a particular metric.

The scores in these correlations were based on rank order data derived from the triadic comparisons by summing the integer scores $(2,1,0)$ across subjects within each group. A rank order of the 105 betweenlandmark distances was thus obtained. For the partial correlational analysis (Kendall's tau), the rank order for each group obtained under euclidean instructions was correlated with the rank order of the ideal euclidean subject. In a similar fashion, the rank order obtained under functional instructions was correlated with that of the perfect functional subject. Because the actual euclidean and functional distances are themselves correlated in this space, as in most ordinary spaces, this intrinsic correlation was partialed out. The resulting partial correlations are presented in Table 1. The top row of Table 1 gives the partial 
Table 1

Partial Rank-Order Correlation Coefficients (Kendall's Tau)

Between Judgments of Subject Groups and Judgments of the Ideal Functional or Euclidean Subject

\begin{tabular}{llccc}
\hline & & \multicolumn{3}{c}{ Group } \\
\cline { 3 - 5 } & & & \multicolumn{3}{c}{$\begin{array}{c}\text { Adventi- } \\
\text { tiously }\end{array}$} & $\begin{array}{c}\text { Congen- } \\
\text { itally } \\
\text { Instruction }\end{array}$ & Baseline & Sighted & Blinded & Blind \\
\hline Neutral & Functional & .64 & .70 & .54 \\
Neutral & Euclidean & .26 & .14 & .31 \\
Functional & Functional & .68 & .73 & .53 \\
Euclidean & Euclidean & .68 & .46 & .31 \\
\hline
\end{tabular}

correlations between judgments obtained under neutral instructions and the judgments of the ideal functional subject. These correlations are relatively high and do not differ markedly from one another. In contrast, the second row shows relatively low partial correlations between judgments obtained under neutral instructions and the ideal euclidean subject judgments. This pattern suggests that when the attention of the subjects is not focused on a particular metric, they more often respond in terms of functional distance than of euclidean distance. This seems to be true regardless of specific visual experience with the space in question or history of visual experience in general.

The third row of Table 1 includes the correlations between judgments under functional instructions and judgments of the ideal functional subject. These are essentially the same as those obtained under neutral instructions. Thus, even under neutral instructions, subjects seem to operate with respect to a functional metric as well as they are able to, even when instructed to do so. A different pattern appears in the fourth row, which shows the correlations of judgments obtained under euclidean instructions with ideal euclidean judgments. Here, the correlations for the sighted and the adventitiously blinded subjects are substantially higher than those obtained under neutral instructions. On the other hand, there is no difference for the congenitally blind subjects. This pattern suggests that the sighted and, possibly to a lesser degree, the adventitiously blinded are flexible in shifting to a euclidean metric when instructed to do so. The congenitally blind subjects, however, do not seem to make any adjustment to such instructions. This pattern is consistent with the results of the euclidean instruction analysis of variance described above. These general results suggest that prior visual experience may make one more flexible in the use of spatial metrics.

\section{Third Analysis: Multidimensional Scaling}

Multidimensional scaling was used to portray visually each group's comparative distance judgments when they were asked to base judgments on euclidean distances. These spatial representations were generated to help relate each group's errors to actual features of the experimental space. KYST, a recent version of nonmetric multidimensional scaling devised by Kruskal, Young, and Seery (Note 1), was used. The computer program uses an iterative procedure, searching for the $\mathrm{n}$-dimensional (in the present case, two-dimensional) configuration of the stimulus locations that best preserves the rank order of input distances. It proceeds by minimizing a goodness-of-fit statistic called "stress," which indicates how closely the input distance data are fit by the distances measurable on the computer-drawn configuration.

The input to the scaling program was the rank orders derived from each group's triadic comparisons under euclidean instruction. The computer-generated spatial configuration derived from each group's judgments fit quite well with those judgments, as indicated by low stress values ranging from .127 to .145 . To clarify the presentation of the computerdrawn spatial representation, the computer program CONGRU (Oliver, Note 2) was used to rotate and adjust the scale size of the configuration derived from each group's judgments until it was maximally congruent with a model configuration, the actual layout of the experimental space. The degree of congruence between the two configurations is indicated by a rootmean-square error term. Each group's spatial solution appears in Figure 2 along with the layout of the experimental space. By inspection of Figure 2, it can be seen that the spatial solution derived from each group's triadic distance comparisons closely resembles the layout of the experimental space. Although each subject made numerous errors in judgment, the errors do not indicate an overall misconception of the spatial layout by any of the groups. It may be that high accuracy of this sort is achieved by the blind only for greatly overlearned spaces of the sort used in the present experiment.

As expected from the previous analyses, the worst fitting solution was derived from the congenitally blind group's judgments and the best fitting was derived from the sighted group's judgments. These differences can be related to perceptible features of the experimental space by inspection of each group's spatial configuration in Figure 2. In terms of the configurations, the blind subjects tended to exaggerate euclidean distances between locations that are functionally separated by corners and turns more than did sighted subjects. To illustrate this point, consider the location of landmark 15 in Figure $2 a$, taken from the blueprint of the experimental space. Locations 15 , 14 , and 13 can be traversed by straight-line routes. On the other hand, travel from 15 to the other landmarks requires turns to negotiate intervening corners. By inspection of Figures $2 \mathrm{~b}, 2 \mathrm{c}$, and $2 \mathrm{~d}$, landmark 15 is farther up on the configuration than it should be according to its location on the blueprint. In psychological terms, this means that 15 was judged by subjects to be closer to 14 and 13 than it really is and 

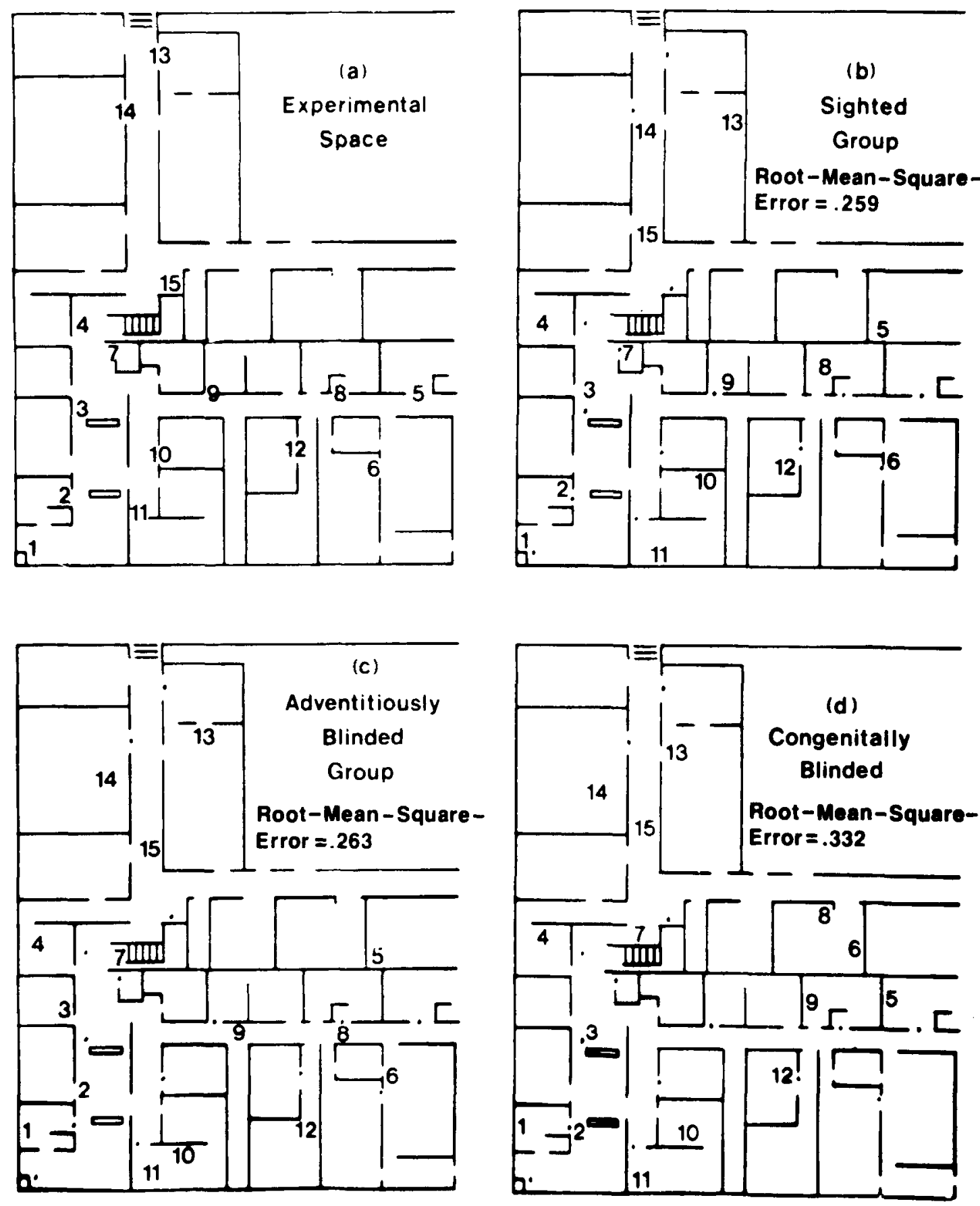

Figure 2. Layout of experimental space and multidimensional scaling solutions for sighted, adventitiously blinded, and congenitally blind groups.

farther from the remaining locations than it really is. Note that the amount of this error is least for the sighted and most for the congenitally blind.

A similar interpretation can be applied to landmark 9. Note from Figure $2 a$ that one can travel a straight line between 9 and many locations on the right-hand side of the figure, whereas direct travel between 9 and left-hand locations is not possible. On the configuration derived for the sighted subjects, 9 is located slightly to the right of its actual location within the space. Again, this error is exaggerated more for the adventitiously blinded and most of all for the congenitally blind.

\section{CONCLUSIONS}

The most impressive result of the present study is the accuracy of the blind subjects' knowledge of this 
large-scale spatial layout. Of course, they were selected for having a good functional knowledge of the space, but the scaling solutions depicted in Figure 2 indicate remarkably good euclidean knowledge as well. In terms of their relative performance, however, the analyses of the euclidean instructions indicate that the sighted group performs best, the adventitiously blinded group is intermediate, and the congenitally blind subjects perform least well. A weakness of the congenitally blind subjects was their apparent difficulty in shifting from a functional to a euclidean basis for their distance judgments, as indicated especially in the partial correlation analysis. The fact that there were differences between the sighted and adventitiously blinded groups as well as between the adventitiously blinded and congenitally blind groups suggests that both specific and general visual experience play roles in representing space. The difference between the sighted and adventitiously blinded groups indicates that specific visual experience with the space used in this study was a factor in the accuracy of their euclidean judgments. The difference between adventitiously blinded subjects and congenitally blind subjects suggests that prior general visual experience is also a factor, a conclusion that is congruent with much of the other literature on the role of visual experience in spatial tasks (Pick, 1974; Warren, Anooshian, \& Bollinger, 1973; however, see Juurmaa, 1973 , for a review that questions the importance of visual experience in spatial tasks).

Previous research comparing the performance of sighted and blind persons has focused on small-scale spatial tasks. When locomotion was involved, the experimental space typically was confined to one room (Worchel, 1951). The present study extends the typical pattern of results to large-scale spaces, with congenitally blind subjects performing worse than adventitiously blinded subjects, even though the performance of the congenitally blind group was relatively accurate.

The use of multidimensional scaling is a promising method for comparing spatial knowledge of sighted and blind subjects. In the present study, the goal was to compare the spatial knowledge of highly experienced and competent members of sighted and blind groups. However, the multidimensional scaling procedures may be particularly useful for assessing knowledge of people as they are acquiring experience in a space. Such a use would have both theoretical and practical implications. For example, different mobility training procedures could be compared or an individual's progress within a mobility training program could be assessed. Such objective procedures are often lacking within orientation and mobility programs (Shingledecker \& Foulke, 1978). In addi- tion, another attractive feature of the multidimensional scaling procedures is that they are also applicable to three-dimensional spaces. No work exists on the role of visual experience in the mental representation of three-dimensional spaces.

\section{REFERENCE NOTES}

1. Kruskal, J. B., Young, F. W., \& Seery, J. B. How to use KYST, a very flexible program to do multidimensional scaling and unfolding. Unpublished manuscript, Bell Laboratories, 1973

2. Oliver, D. Metrics for comparison of multidimensional scalings. Unpublished manuscript, Harvard University, 1970.

\section{REFERENCES}

Attneave, F., \& Farrar, P. The visual world behind the head. American Journal of Psychology, 1977, 90, 549-563.

Gibson, E. J., \& Walk, R. D. The "visual cliff." Scientific American, 1960, 202, 64-71.

Gregory, P. L., \& Waldace, J. G. Recovery from early hlindness: $A$ case study (Monograph No. 2). Cambridge: Experimental Psychology Society, 1963.

Hazen, N. L., Lockman, J. J., \& Pıск, H. L., J h. The development of children's representations of large-scale environments. Child Development, 1978, 49, 623-636.

Невв. D. O. The innate organization of visual activity. 1. Perception of figures by rats reared in total darkness. Journal of Genetic Psychology, 1937, 51, 101-126.

Hein, A., \& Diamond, R. M. Locomotory space as a prerequisite for acquiring visually guided reaching in kittens. Journal of Comparative and Physiological Psychology, 1972, 81, 394-398.

JuurmaA, J. Transposition in mental spatial manipulation: A theoretical analysis. American Foundation for the Blind Research Bulletin, 1973, 26, 87-134.

Levelt, W. J., Van de Geer, J. P., \& Plomp. R. Triadic comparisons of musical intervals. British Journal of Mathematical and Statistical Psychology, 1966, 19, 163-179.

Plck, H. L., Jr. Visual coding of nonvisual spatial information. In R. B. MacLeod \& H. L. Pick, Jr. (Eds.), Perception: Essays in honor of J. J. Gibson. Ithaca, N.Y: Cornell University Press, 1974.

Shingledecker, C. A., \& Foulke, E. A human factors approach to the assessment of the mobility of blind pedestrians. Human Factors, 1978, 20, 273-286.

Sifiel, A. W., \& White, S. H. The development of spatial representations of large-scale environments. In $\mathrm{H}$. W. Reese (Ed.), Advances in child development and behavior (Vol. 10). New York: Academic Press, 1975.

Warren, D. H., Anooshian, L. J., \& Boldinger, J. G. Early vs. late blindness: The role of early vision in spatial behavior. American Foundation for the Blind Research Bulletin. 1973, 26. 151-170.

Worrhls, P. Space perception and orientation in the blind. Psychological Monographs, 1951, 65, 1-28.

\section{NOTES}

1. Use of an incomplete subset of triads introduces a measurable amount of experimental error into our measures. This experimental error does not invalidate between-group comparisons, since sub. jects were all tested on the same set of triads.

(Received for publication February 8, 1980; revision accepted June 16, 1980.) 